\title{
The effectiveness of a pile barrier for vibration transmission in a soil stratum over a rigid bedrock*
}

\author{
Guillermo M. Álamo ${ }^{1, *}$, Jacob D.R. Bordón ${ }^{1}$, Juan J. Aznárez ${ }^{1}$, Geert Lombaert ${ }^{2}$ \\ ${ }^{1}$ Insituto Universitario de Sistemas Inteligentes y Aplicaciones Numéricas en Ingeniería (SIANI), \\ Universidad de Las Palmas de Gran Canaria, Edificio Central del Parque Científico y Tecnológico, \\ Campus Universitario de Tafira 35017 Las Palmas de Gran Canaria, Spain \\ guillermo.alamo / jacobdavid.rodriguezbordon / juanjose.aznarez @ulpgc.es \\ 2 Department of Civil Engineering, KU Leuven, Kasteelpark Arenberg 40, B-3001 Leuven, Belgium \\ geert.lombaert@kuleuven.be
}

\begin{abstract}
The potential use of pile barriers as a ground vibration mitigation measure is analysed through an efficient numerical model. The soil is modelled as an elastic half space, using the reciprocity theorem and specific Green's functions for the layered half space, while the piles are treated as beam elements. After benchmarking the proposed model against a Boundary Element model, it is used to study this type of barrier, with particular focus on how the presence of a rigid bedrock alters its performance. A significantly different behaviour when compared to the case of the half space is found if the piles reach the rigid stratum, increasing the barrier performance around the layer second natural frequency.
\end{abstract}

Keywords: Vibration isolation, Pile barriers, Elastic wave propagation, Soil stratum, Boundary integral formulation

\section{Introduction}

The environmental vibration produced by nearby traffic, machines or construction operations can lead to annoyance for residents or perturbed operation of sensitive equipment. Different types of isolation systems can be used to attenuate these ground vibrations, located either at the source point or at the receiver structure. In addition to these, wave barriers can be used along the transmission path to reduce the propagation of vibrations in the ground.

Several types of wave barriers have been used and studied along the last decades. The simplest system consists of an open or in-filled trench situated between the vibration

*Post-print of the paper published in COMPUTERS AND GEOTECHNICS, 110, 274-286 (2019), DOI: $10.1016 /$ j.compgeo.2019.02.022 
source and the receiver point. The surface disturbance imposed by the trench diffracts the elastic waves resulting in a vibration amplitude reduction [1]. The effectiveness of trenches is determined by their depth and the soil wavelength [1-3] and, for open or in-filled, has been the object of study of a large variety of research using an either experimental [35] or numerical [6-12] approach. Depending on the dimensions of the trench or the characteristics of the soil, sometimes it is necessary to include structural elements, such as thin shell walls [13] or sheet-pile walls [14 16], to guarantee its stability. Systems composed of double wall barriers have been also recently considered [17, 18]. Some of these works [12, 18] have employed optimization techniques in order to improve the performance of the barriers.

On the other hand, wave barriers formed by piles can be also used to mitigate the ground vibrations when large barrier depths are required. The pioneering experimental work of Woods et al. [19] and Liao and Sangrey [20], and the analyses based on the equations of the problem of Avilés and Sánchez-Sesma [21, 22] considered piles for impeding vibration transmission. Kattis et al. [23] used a Boundary Element (BE) formulation to analyse the performance of a row of piles to mitigate the ground surface vibrations produced by an external vertical load. In later work, they addressed the problem by modelling the pile barrier through an effective trench [24]. A BE model was also used by Tsai et al. [25] to study the effectiveness of barriers formed by different types of hollow and solid piles. Configurations that differ from a single row of piles were considered by Gao et al. [26] who studied the performance of three pile rows to mitigate Rayleigh waves; while Xia et al. 27] proposed a formulation to analyse the scattering effects of an arbitrary configuration of piles. Also, pile barriers with elements disposed in a periodic configuration (periodic pile barriers) have been analysed in several studies [28 30]. Some authors have also addressed the problem of pile barriers embedded in poroelastic soils 31 33].

At this point, it should be mentioned other novel systems that have been proposed recently in order to mitigate the vibrations received by structures. One example is the application of structure-soil-structure interaction effects [34, 35] through an auxiliary system that absorbs part of the energy transmitted through the ground. Also, different kinds of metabarriers or metamaterials [36 42], some of them based on optical principles, can be used to properly channel or filter the elastic waves and, consequently, reduce the ground vibrations at the desired locations.

In most of the previous work, the piles were assumed to be in a homogeneous half space. There are few works which study how the soil stratification affects the performance of the pile barrier (see e.g. [43]). In order to fill this gap, this work analyses the behaviour of pile barriers for both the case of a half space and the case of a layer on bedrock site. The study is performed in the frequency domain by a numerical model based on the integral expression of the reciprocity theorem and the use of fundamental solutions for the layered half space. In this formulation, piles are treated as load lines and coupled to the soil using a Timoshenko beam model.

The document is organized as follows. First, the problem under study is defined in Section 2 and the formulation of the proposed model is presented in Section 3 , Then, the ability of the integral model to simulate the effects that are involved in the pile barrier problem is validated by comparing with a Boundary Element model in Section 4. After 
setting the scope of applicability of the numerical tool, results are presented in Section 5 , followed by the main conclusions drawn from them in Section 6 .

\section{Problem statement}

The effectiveness of a row of identical piles for the mitigation of ground vibrations is studied first. A point load in vertical direction, centrally placed at one side of the pile barrier, is assumed as the source of vibrations. On the other side of the barrier, the response at a set of observation (receiver) points uniformly distributed along a rectangular surface behind the barrier is measured. Other choices like taking an area relative to a wavelength (typically the Rayleigh wavelength) would have resulted in an over-complicated problem definition given the number of parameters involved (including non-homogeneous soils). Instead, by choosing a fixed area, results become concise and give an insight on the effects of the barrier over its surroundings. A sketch of the problem is shown in Fig. 1.

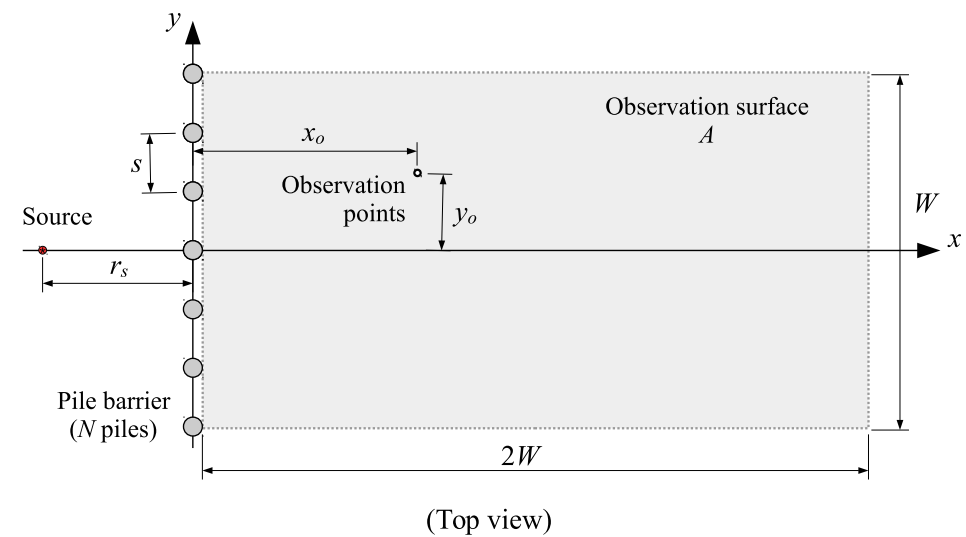

Figure 1: Sketch of the studied problem.

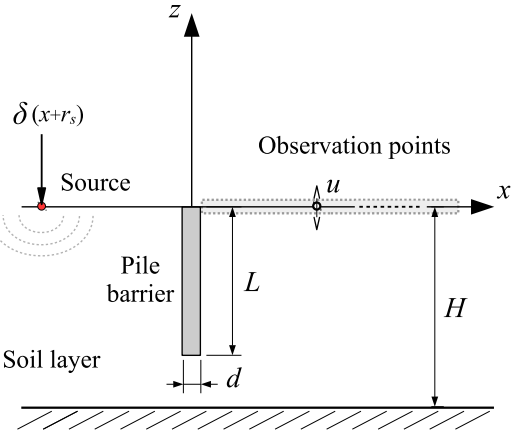

Bedrock (Plane view)

In order to present results that can be applied to a wide set of scenarios, the problem is defined in terms of dimensionless parameters. The pile geometry is determined by the pile aspect ratio $L / d$; while the barrier geometry is given by the number of piles $N$ and centreto-centre pile separation $s / d$. The width of the barrier is denoted as $W / d=(N-1)(s / d)$.

The source is placed at the central line of the barrier at a dimensionless distance $r_{s} / d$ in the negative $x$ direction. On the other hand, the position of each observation point is defined through the parameters $x_{o} / d$ and $y_{o} / d$ corresponding to the coordinates of the points in the reference system located at the centre of the barrier.

The work at hand aims to study how the performance of the pile barrier is affected by the presence of a stiff bedrock at the site. Therefore, two soil profiles are considered: a half space and a single soil layer over a bedrock. The relative stiffness between the soil (half space or upper layer) and the pile is given by the pile-soil Young's modulus ratio $E_{p} / E_{s}$. For the soil deposit profile, the thickness of the layer is given by the ratio $H / d$, and the relative stiffness between bedrock and the upper layer is determined by the ratio $E_{b} / E_{s}$. The other soil properties are equal for the layer, bedrock and half space and are: soil density $\rho_{s}$ (soil-pile density ratio $\rho_{s} / \rho_{p}$ ), soil Poisson's ratio $\nu_{s}$ and soil hysteretic 
damping ratio $\beta_{s}$, which gives complex valued elastic properties as $E^{*}=\operatorname{Real}[E]\left(1+2 \mathrm{i} \beta_{s}\right)$, with $\mathrm{i}$ being the imaginary unit.

The remaining material properties corresponding to the piles are: pile Poisson's ratio $\nu_{p}$ and pile hysteretic damping ratio $\beta_{p}$.

Results are presented in terms of the dimensionless frequency $a_{0}=f d / c_{s}$, being $f$ the excitation frequency in hertz and $c_{s}$ the shear wave propagation velocity of the soil (half space or upper layer). The dimensionless frequency represents the ratio between the pile diameter and the shear wave wavelength in the soil, i.e., $a_{0}=d / \lambda_{s}$. Other definitions are possible, e.g. using the pile length as the reference length. However, as it is shown in Section 4, the use of the pile diameter as the reference length allows to establish a validity range of the proposed model independent of pile dimensions.

The reduction of surface displacements produced by the pile barrier is measured in terms of the amplification ratio:

$$
A_{r}=\left|\frac{u}{u_{\text {ref }}}\right|
$$

where $u$ and $u_{\text {ref }}$ are the vertical displacements produced at each observation point by the vertical load with or without the presence of the pile barrier, respectively.

Then, the effectiveness of the pile barrier is measured through the average amplification ratio along the observation surface, which is obtained as:

$$
\overline{A_{r}}=\frac{1}{A} \int_{A} A_{r} \mathrm{~d} A
$$

being $A=2(W / d)^{2}$ the dimensionless area of the rectangular observation surface located behind the pile barrier. As mentioned before, in order to evaluate the two-dimensional integral presented in Eq. (2), the observation surface is discretized into elements whose response is determined by the one of the corresponding observation point. These observation points are distributed over the observation area $A$ with a spacing equal to the pile diameter both in the $x$ and $y$ directions for all the studied frequencies.

\section{Methodology}

The response at the observation points due to the point load is computed through a three-dimensional time-harmonic numerical model previously developed by the authors 444] for the dynamic analysis of pile foundations. In general, the model assumes the soil as a finite number of zoned homogeneous viscoelastic isotropic layers overlying a homogeneous half space. Its behaviour is represented through the reciprocity theorem in elastodynamics in combination with specific Green's functions for the layered half space [45]. In the soil formulation, the piles are treated as load lines and the pile-soil interaction is expressed through tractions acting in the three directions of the space. Piles are modelled as Timoshenko beam finite elements and coupled with the soil by imposing compatibility and equilibrium conditions over the pile/soil displacements and the soil-pile interaction tractions. The use of the specific Green's functions (defined as the response of the layered half space to an unitary point load applied at each direction of the three-dimensional space) as fundamental solution and the treatment of piles as load lines (therefore, not affecting the soil continuity), results in a very efficient model 
as it completely avoids the meshing of the free-surface or layer interfaces, obtaining a formulation only in terms of pile variables. Once the response of the piles is known, the displacements at the soil observation points are obtained by the application of the reciprocity theorem at these internal points. In the following, a brief summary of the model is presented, which is supported by the graphical description presented in Fig. 2 .

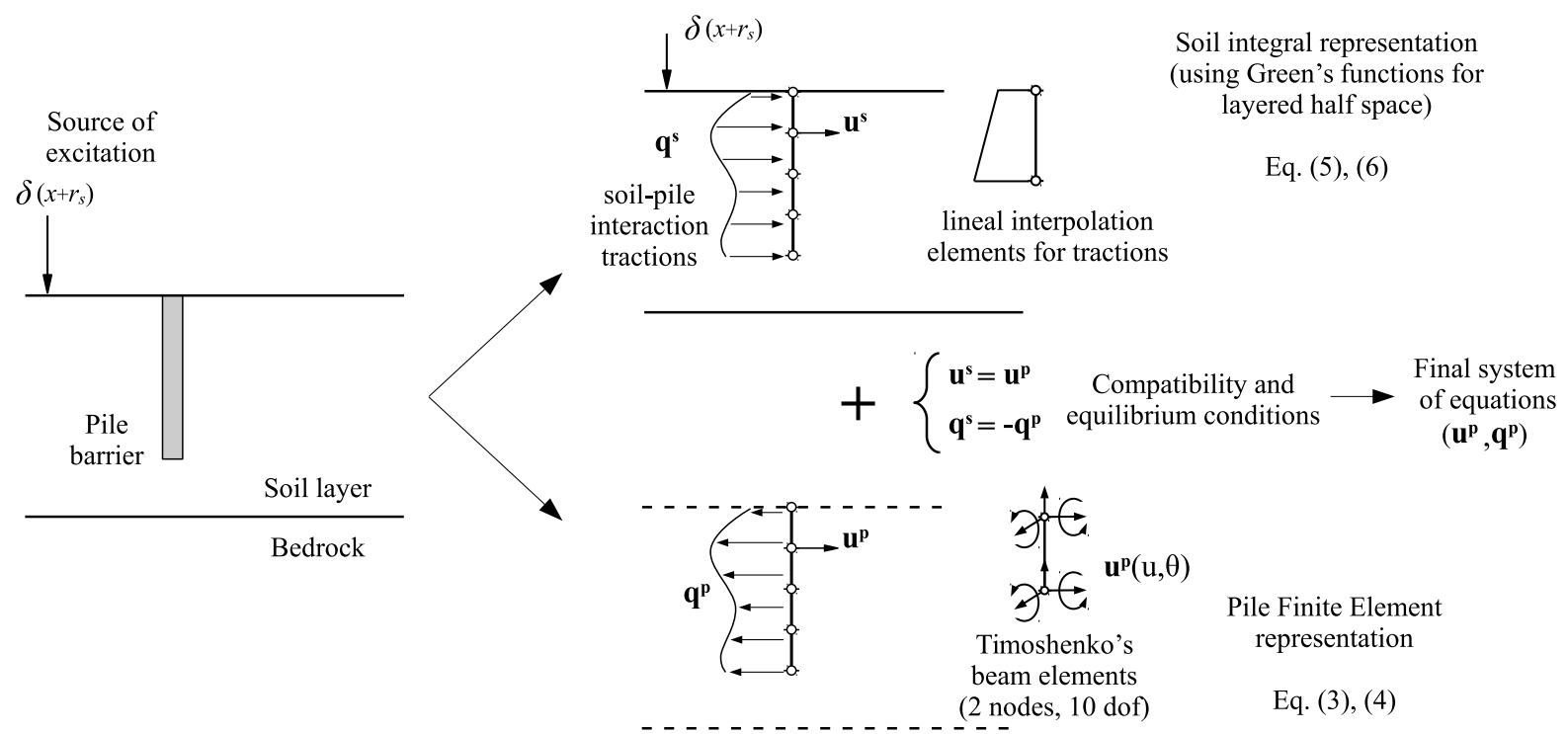

Figure 2: Sketch of the proposed model for the analysis of pile barriers.

Piles. The dynamic behaviour of the piles is represented through two-noded Timoshenko beam elements with 8 unknowns per node corresponding to three displacements, two bending rotations, and three soil-pile interaction tractions. The torsional motion of the pile is neglected as no interaction with the soil is considered in the proposed model for this specific mode. The pile bending deformation is modelled inside each element through cubic (for displacements) and quadratic (for rotations) shape functions that satisfy the static equation of the Timoshenko beam. The formulation of this FE element (shape functions and elemental matrices) can be found in [46]. On the other hand, linear shape functions are used for the axial displacements and for the soil-pile interaction tractions along each element. Assuming time-harmonic displacements and forces, the system of finite element equations for the piles can be expressed as:

$$
\left(\mathbf{K}\left(1+2 \mathrm{i} \beta_{p}\right)-(2 \pi f)^{2} \mathbf{M}\right) \mathbf{u}^{\mathbf{p}}-\mathbf{Q} \mathbf{q}^{\mathbf{p}}=\mathbf{0}
$$

where $\mathbf{K}$ and $\mathbf{M}$ are the global stiffness and mass matrices obtained from the assembly of the elemental ones, $\mathbf{Q}$ is the global matrix that transforms the distributed tractions into the corresponding nodal loads, and $\mathbf{u}^{\mathbf{p}}$ and $\mathbf{q}^{\mathbf{p}}$ are the vectors of nodal displacements (and rotations) and soil-pile interaction tractions of the piles.

The expression given above corresponds to the case of floating piles (free-tip conditions). However, when piles reach a stiff medium, hinged (restricted displacements) or fixed (restricted displacements and rotations) tip conditions can be directly imposed. In order to apply these conditions, Eq. (3) is rearranged so the tip displacements and/or 
rotations vanish and the corresponding reaction forces or moments are included as complementary unknowns. In general, the pile system of equations can be written as:

$$
\left(\mathbf{K}\left(1+2 \mathrm{i} \beta_{p}\right)-(2 \pi f)^{2} \mathbf{M}\right) \mathbf{u}^{\mathbf{p}}-\mathbf{Q q}^{\mathbf{p}}-\mathbf{F}^{\mathrm{tip}}=\mathbf{0}
$$

where $\mathbf{F}^{\text {tip }}$ is the vector containing the tip reaction forces and/or moments acting over the pile due to the imposed conditions.

Soil. The treatment of piles as load lines, the boundary conditions of the studied problem and the layered half space Green's functions simplify the integral expression of the reciprocity theorem in elastodynamics to:

$$
\mathbf{u}^{\kappa}=\sum_{l=1}^{N} \int_{\Gamma_{l}} \mathbf{q}_{l}^{\mathbf{s}} \mathbf{u}^{*} \mathrm{~d} \Gamma_{l}+\mathbf{u}^{*} \mathbf{F}
$$

where $\mathbf{u}^{\kappa}$ is the vector containing the three displacements at the collocation point $\kappa, \Gamma_{l}$ denotes the load line corresponding to pile $l, \mathbf{u}^{*}$ is the tensor containing the displacement Green's functions, $\mathbf{q}_{l}^{\mathbf{s}}$ are the soil-pile interaction tractions acting over the soil corresponding to pile $l$, and $\mathbf{F}=\{0,0,1\}^{\mathrm{T}}$ is the vector containing the external point load (source) acting over the free-surface.

Applying the integral equation (5) to all pile nodes, the system of equations corresponding to the soil is obtained as:

$$
\Upsilon \mathbf{u}^{\mathrm{s}}-\mathrm{Gq}^{\mathrm{s}}=\hat{\mathbf{F}}
$$

where $\mathbf{u}^{\mathbf{s}}$ and $\mathbf{q}^{\mathbf{s}}$ are the vectors containing the soil displacements and soil interaction tractions at all pile nodes, $\Upsilon$ is the collocation matrix which contains either identity submatrices or the corresponding pile displacements shape functions depending on whether or not a non-nodal collocation strategy is needed (see [44]), G is the influence matrix obtained by integrating the fundamental solution displacements times the linear shape functions of the interaction tractions along each pile element, and $\hat{\mathbf{F}}$ is the vector containing the excitation load value times the corresponding displacement fundamental solution terms.

Soil-pile model. As indicated before, the coupling between the pile and soil is realized by imposing compatibility conditions in terms of the displacements at the pile nodes $\left(\mathbf{u}^{\mathbf{s}}=\mathbf{u}^{\mathbf{p}}\right)$ and equilibrium conditions for the soil-pile interaction tractions $\left(\mathbf{q}^{\mathbf{s}}=-\mathbf{q}^{\mathbf{p}}\right)$, obtaining a final linear system of equations that, once solved, gives the nodal values of the pile displacements and interaction tractions.

At a post-processing step, the displacement vector $\mathbf{u}^{i}$ at any internal point $i$ of the soil domain (including free surface) can be computed by applying the integral equation (5) assuming the point $i$ as the collocation point. This equation can be rewritten in matrix form as:

$$
\mathbf{u}^{i}=-\mathbf{G}^{i} \mathbf{q}^{\mathbf{p}}+\mathbf{u}^{*} \mathbf{F}
$$

where $\mathbf{G}^{i}$ is the corresponding influence matrix.

Note that the layered half space Green's functions can also be used to directly compute the reference soil displacement (without barrier) $\mathbf{u}_{\mathrm{ref}}^{i}$ at the internal point $i$. 


\section{Validation of the proposed model. Scope of appli- cability}

The aim of this section is to validate the proposed formulation for application to the pile barrier problem. The presence of the barrier mitigates the ground vibration due to the stiffening of the soil between the receiver and the source points and due to the diffraction of the travelling waves that transmit the energy introduced by the external force. Part of these diffraction effects corresponds to geometrical reflections and transmissions that are produced at the soil-pile interface. However, as the proposed formulation treats the piles as unidimensional beam elements, these phenomena can not be completely captured by the present method. The importance of the geometrical diffraction is expected to increase as the frequency of the excitation augments, when the soil wavelengths are comparable to the pile cross-section dimensions.

Therefore, in order to test the range of applicability of the proposed model, a reduced set of problems is considered and the results of the integral model are compared with the ones of a previously developed continuum-based Boundary Element formulation [47, 48]. The use of this kind of BE models to rigorously handle the pile barrier mitigation problems can be found in the literature (see e.g., [23, 25]). However, this kind of models demands high computational resources (both in time and memory) and also presents, in their standard formulations, the uncertainties intrinsically related to the surface and pile meshing. Those inconveniences are avoided by the proposed integral model, which makes it a suitable tool for carrying out parametric analyses if the validation process is satisfactorily completed.

For the validation analysis, several configurations of pile barriers embedded in a homogeneous half space domain are considered by combining the following dimensionless parameters:

- pile aspect ratio $L / d=10$ and 20

- pile separation distance $s / d=1.5,2,3,4$ and 5

- pile-soil Young's modulus ratio $E_{p} / E_{s}=65$ and 250

- soil Poisson's ratio $\nu_{s}=0.3$ and 0.485

All other dimensionless parameters are kept constant: number of piles $N=7$, pile Poisson's ratio $\nu_{p}=0.25$, pile hysteretic damping ratio $\beta_{p}=0 \%$, soil hysteretic damping ratio $\beta_{s}=2.5 \%$, soil-pile density ratio $\rho_{s} / \rho_{p}=0.7$. The source load is located at $r_{s} / d=10$ and the response at receivers situated at $x_{o} / d=6,10,15$ and 20 and at $y_{o} / d=0$ and $1.5 \mathrm{~s} / \mathrm{d}$ from the centre of the pile barrier are considered. Values of the amplification ratio $A_{r}$ at these observation points are obtained for a frequency range from $a_{0}=0$ to 0.25 .

It is important to highlight that the validation is made in terms of the amplification ratio at specific points instead of using its average value along the whole observation surface. A compensation of errors in the average trend could mask the mismatch between the responses obtained by the proposed and BE models.

For illustration purposes, Fig. 3 shows the meshes required for solving one of the studied configurations by the BE and integral models. Note that only one-quarter of 

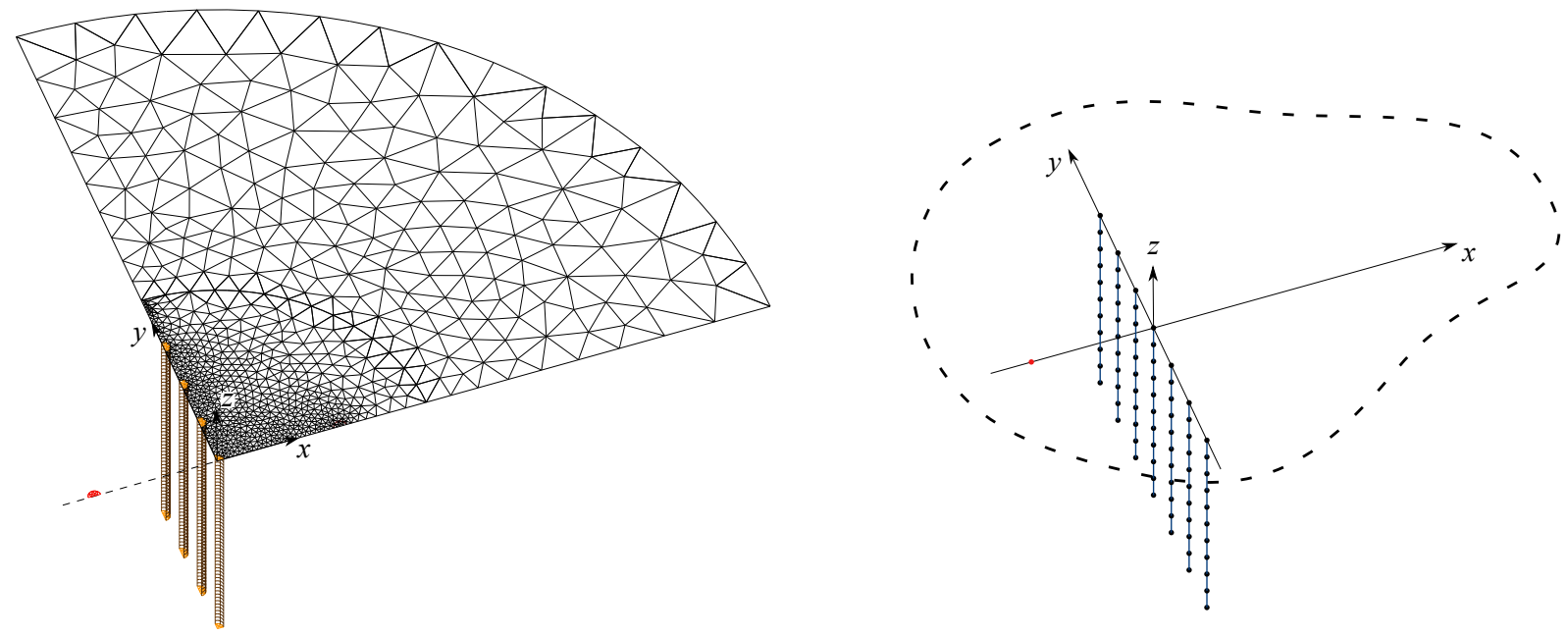

Figure 3: Examples of the meshes used for the BE (left) and integral (right) models.

the discretization of the problem is presented for the BE model in order to clearly show its details. In fact, in order to reduce the computational cost related to solving the corresponding system of equations, the BE model exploits the symmetry of the system, decomposing the problem into its symmetric and antisymmetric components. The computational efficiency of the integral model makes this decomposition unnecessary. Thus, the proposed model directly considers the whole configuration (as shown in Fig. 3). In order to situate the computational performance of both models, wall clock time and RAM memory consumption required for solving one of the considered configurations $(L / d=20$, $s / d=3, E_{p} / E_{s}=65$ and $\left.\nu_{s}=0.485\right)$ are presented. The BE model takes $210 \mathrm{~s}$ per frequency with a memory usage of $11.6 \mathrm{~GB}$ for solving a model of one symmetric quarter of the problem. On the other hand, the integral model takes $5.7 \mathrm{~s}$ per frequency with a memory usage of $110 \mathrm{MB}$ for solving the complete problem. This means that the integral model just takes $2.7 \%$ of the time and $0.9 \%$ of the memory required by the BE model. These values were obtained by using a 28 core (Intel ${ }^{\circledR}$ Xeon ${ }^{\circledR}$ CPU E5-2690 v4 (a 2.60GHz) 260GB RAM computer.

Fig. 4 presents an example of the comparison between the results of the two models. The amplification ratio $A_{r}$ at the observation points defined before that are obtained by the integral and the BE models are plotted against the dimensionless frequency. Configurations of $L / d=20$ and $\nu_{s}=0.485$ are considered. The results corresponding to the different pile separation distances are shown in different rows, while the observation points are indicated by the value of $x_{o} / d$ at the top of each column. It can be seen that, depending on the pile configuration, the range of applicability of the propose model changes. In order to determine this in an objective way, a simple statistical analysis of the data is conducted, which is detailed in the following.

The combination of the aforementioned parameters results in 320 curves of the evolution of the amplification ratio $A_{r}$ with the dimensionless frequency that can be used to compare the two different methodologies. In order to measure the discrepancies between the two models at any point $\mathbf{x}_{\mathbf{o}}=\left\{x_{o} / d, y_{o} / d, 0\right\}^{\mathrm{T}}$ of the soil surface, the following 

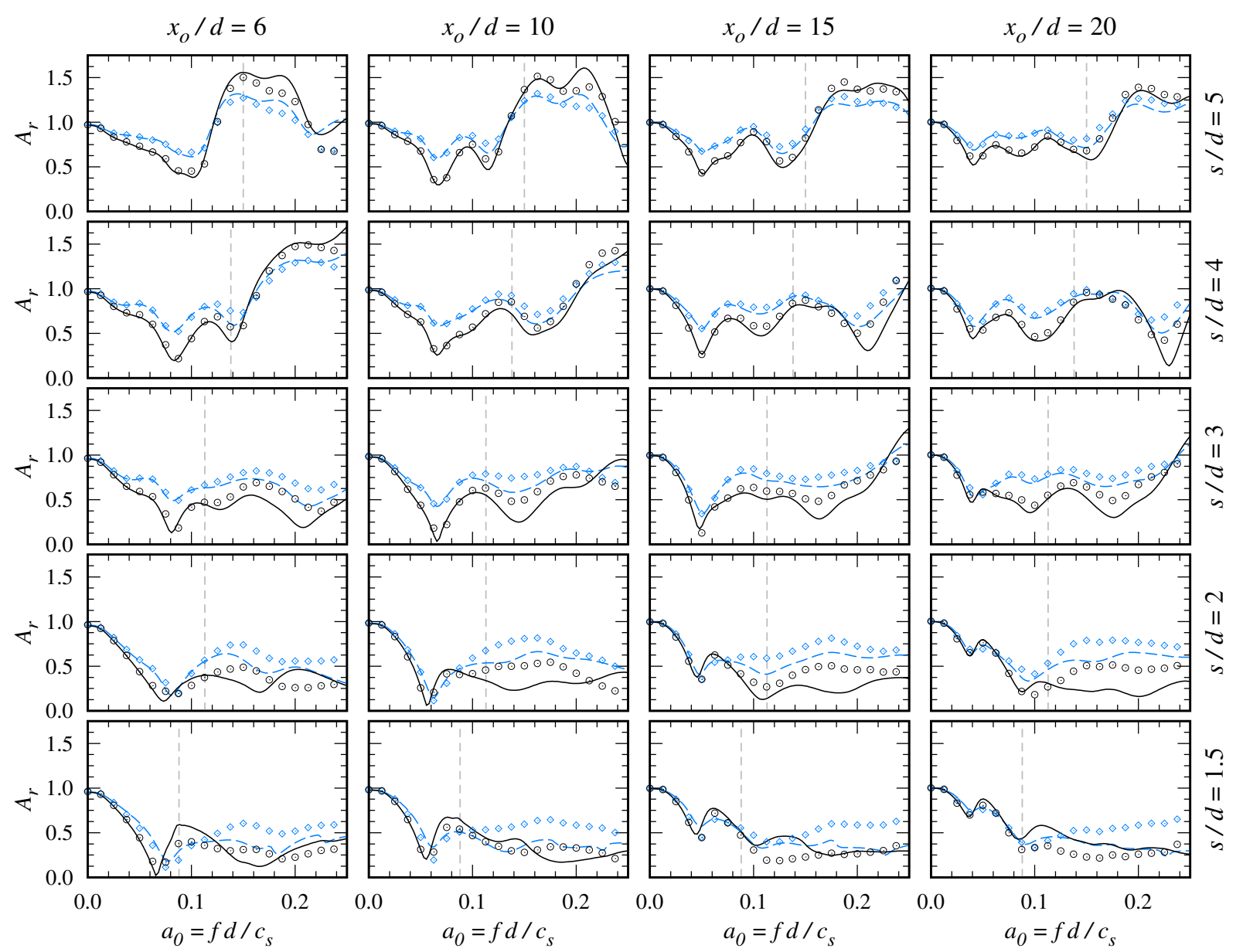

Figure 4: Comparison between amplification ratios obtained by the integral (lines) and BE (markers) models. $\nu_{s}=0.485, L / d=20, E_{p} / E_{s}=65$ (blue) or 250 (black). Observation points located at the centre of the barrier $\left(y_{o} / d=0\right)$.

frequency-dependent relative error is defined:

$$
\Delta_{A_{r}}\left(a_{0}, \mathbf{x}_{\mathbf{o}}\right)=\left|\frac{A_{r}\left(a_{0}, \mathbf{x}_{\mathbf{o}}\right)-A_{r}^{\mathrm{BE}}\left(a_{0}, \mathbf{x}_{\mathbf{o}}\right)}{\max _{a_{0}}\left(A_{r}^{\mathrm{BE}}\right)-\min _{a_{0}}\left(A_{r}^{\mathrm{BE}}\right)}\right|
$$

which represents the importance of the difference between the amplification ratio obtained by the integral $\left(A_{r}\left(a_{0}, \mathbf{x}_{\mathbf{o}}\right)\right)$ and $\mathrm{BE}\left(A_{r}^{\mathrm{BE}}\left(a_{0}, \mathbf{x}_{\mathbf{o}}\right)\right)$ models with respect to the range within which lies the reference (BE) results. This definition of the relative difference is chosen over the classic relative error in order to avoid excessive values at frequencies in which the reference value of $A_{r}^{\mathrm{BE}}$ is close to zero.

After computing the Pearson's correlation coefficient $(R)$ between $\Delta_{A_{r}}$ and the variable dimensionless parameters, the only ones that are found to be significantly related to the differences between the two models are the dimensionless frequency $a_{0}(R=0.614)$ and the separation distance $s / d(R=-0.183)$. As expected, as the frequency increases and, consequently, the soil wavelength decreases, the geometrical effects of the pile dimensions become more important and, therefore, the simplified model is unable to reproduce these 
effects which are taken into account by the BE model. Related to this, as the piles in the barrier are located at closer distances, the volume of soil situated in between the piles differs more from one model to another. Note that, in the limit scenario of $s / d=1$, the piles will touch each other at their perimeters in the BE model, while in the integral model the propagation of waves through that soil medium will be considered. In other words, it is found that as the geometry gets closer to the one assumed in the integral model (unidimensional load lines, i.e., slender separated piles), the results of the two methodologies converge.

Through box and whiskers diagrams, Fig. 5 shows the relation between $\Delta_{A_{r}}$ and the aforementioned relevant parameters $\left(s / d, a_{0}\right)$. In coherence with the correlation analysis, it is found that the mean values of the differences are larger for the smallest pile separation distances, and that the magnitude of these differences significantly increases with the frequency. The results presented in Fig. 5 also indicate the high dispersion that exists for the differences between the models, which lead to the statistical treatment of the data instead of a direct evaluation from the graphical results.

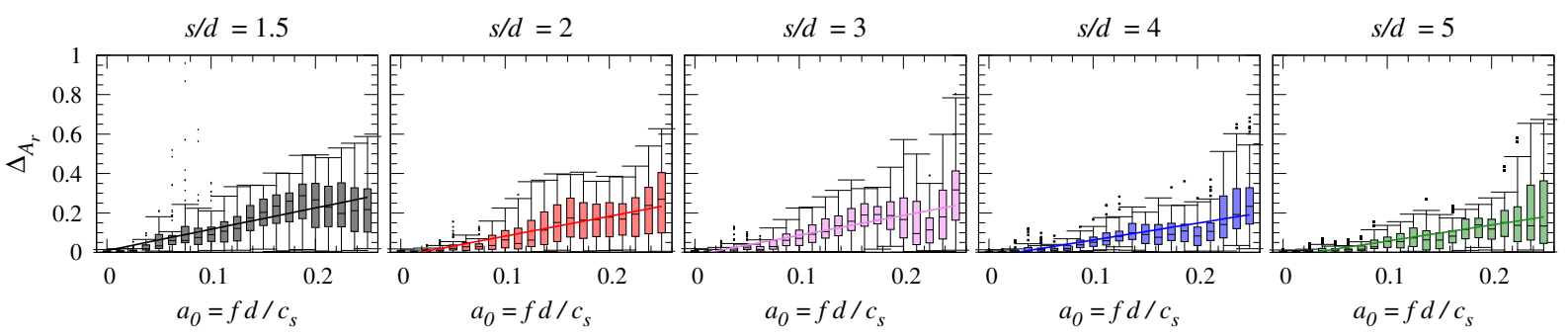

Figure 5: Distribution of the differences $\Delta_{A_{r}}$ between the integral and BE models.

Finally, in order to fix the range of applicability of the integral model, the expected difference $\Delta_{A_{r}}^{*}$ is obtained for each separation distance $s / d$ through a linear regression of the available data and is shown as a solid line in each graphical area of Fig. 5. The comparison between the evolution with frequency of this expected difference for the different separation distances can be easily analysed in Fig. 6(a). From these results, the validity of the proposed model can be established by defining the range in which the discrepancies are acceptable. Obviously, the chosen threshold will depend on the characteristics of the problem under study. For this work, a value of 0.1 (ten percent of the $A_{r}^{\mathrm{BE}}$ range) is assumed in order to accept the results of the integral model, yielding the maximum values of the dimensionless frequencies $a_{0}^{\lim }$ that can be reached for each separation distance, Fig. 6(b). Note that a near-linear relation between the separation distance $s / d$ and the maximum dimensionless frequency $a_{0}^{\mathrm{lim}}$ is obtained. Thus, for separation distances $s / d>5$ and dimensionless frequencies $a_{0}<0.15$ the results of the integral model can be accepted and, therefore, it is the range of properties that is considered in the following analyses.

The different values of maximum dimensionless frequency $a_{0}^{\lim }$ are also indicated as dashed vertical lines in Fig. 4. It can be seen that a good match between the two models is obtained for low frequencies up to the $a_{0}^{\mathrm{lim}}$. Also, for configurations with large pile separation distances, the integral model follows the main trend of the BE results for frequencies larger than $a_{0}^{\text {lim }}$, although their values do not perfectly coincide. Despite this validation study has been presented only for the half space domain, the same conclusions 
(a)

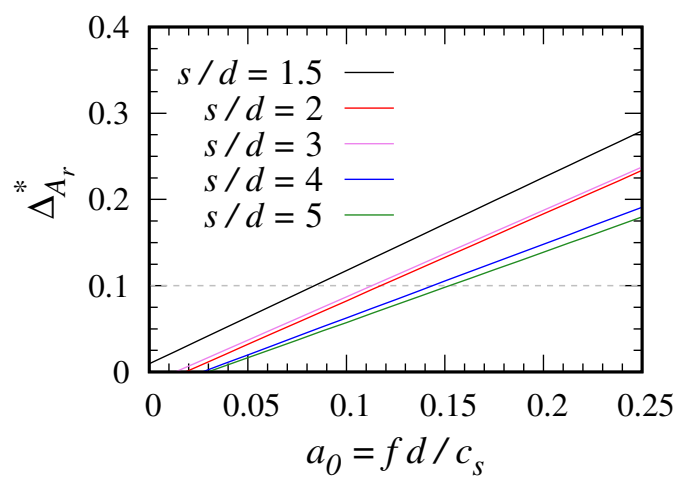

(b)

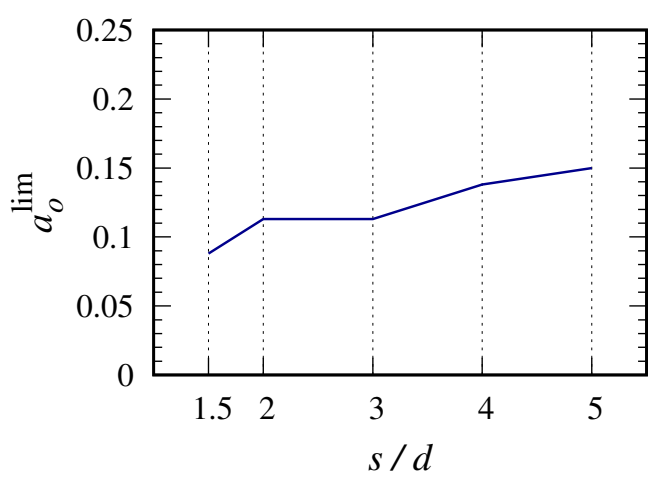

Figure 6: Range of applicability of the integral model. (a): Expected difference $\Delta_{A_{r}}^{*}$ between the integral and BE models. (b): Maximum dimensionless frequency in which $\Delta_{A_{r}}^{*}<0.1$ as function of the pile separation distance $s / d$.

were found when considering the same geometry and properties as the ones using in Fig. 4, but with an infinitely rigid bedrock placed at a depth equal to the pile length. As indicated at the beginning of this section, the discrepancies between the two models are produced due to the lack of geometrical diffraction effects in the integral formulation and these phenomena were expected to behave in a similar way regardless the existence of the rigid bedrock.

\section{Results}

\subsection{Scope of the study}

As previously stated, the objective of the present work is to study how the vibration mitigation performance of the pile barrier is altered when it is embedded in a soil layer over a stiff bedrock. For this purpose, the results corresponding to this profile (henceforth referred to as Bedrock profile) are compared with the ones obtained by assuming the soil as an unbounded semi-infinite medium (Half space profile).

In the next subsections, several cases are considered in order to analyse the effects of some of the dimensionless parameters that define the problem. Table 1 presents each scenario together with the values that are assumed for the different dimensionless parameters and contact condition at the pile tip-bedrock union.

In addition to this, Table 2 shows the values for the rest of the dimensionless properties that are kept constant along the different cases. The chosen value of the soil Poisson's ratio $\nu_{s}=0.485$ corresponds to a real soil profile extracted from [10]. In this regard, as the Poisson's ratio directly affects the propagation of elastic waves in the soil media, it also influences the performance of the pile barrier. On the other hand, the importance of the soil density was found to be marginal after obtaining virtually the same results when varying the density ratio $\rho_{s} / \rho_{p}$ from 0.3 to 2 . Thus, a constant value $\rho_{s} / \rho_{p}=0.7$ presented in Table 2 is considered in this work.

The cases presented allow comparing the performance for vibration mitigation of the 


\begin{tabular}{cccccccc}
\hline Case & $N(s / d)$ & $L / d$ & $H / d$ & $r_{s} / d$ & $E_{p} / E_{s}$ & $E_{b} / E_{s}$ & tip cond. $^{\mathrm{a}}$ \\
\hline $\mathrm{A}$ & $7(4)$ & $5,10,20$ & 20 & 24 & $100,250,500$ & $\infty$ & free \\
$\mathrm{B}$ & $7(4)$ & 10,20 & $L / d$ & 24 & 250 & $\infty$ & free,hinged,fixed \\
$\mathrm{C}$ & $7(4)$ & 10,20 & $L / d$ & $0-6\left(r_{s} / \lambda_{s}\right)$ & 250 & $\infty$ & fixed \\
$\mathrm{D}$ & $7(4)$ & 10,20 & $L / d$ & 24 & 250 & $1,2,5,10, \infty$ & free \\
$\mathrm{E}$ & $7(4)$ & 10,20 & $L / d$ & 24 & $250^{\mathrm{c}}$ & $\infty$ & fixed \\
$\mathrm{F}$ & $\mathrm{b}$ & 10,20 & $L / d$ & 24 & 250 & $\infty$ & fixed \\
\hline
\end{tabular}

${ }^{a}$ only for the Bedrock scenario. Free tip conditions are always assumed for the half space profile.

${ }^{\mathrm{b}}$ single barrier configurations: 13(2),7(4),4(8); double barrier configurations: $6 \& 7(4), 3 \& 4(8)$.

${ }^{\mathrm{c}}$ non-homogeneous stratum (Gibson soil). $E_{p} / E_{s}$ indicates the average value of the layer.

Table 1: Summary table with the definition of the cases used in this study.

\begin{tabular}{cccccc}
\hline$\rho_{s} / \rho_{p}$ & $\nu_{s}$ & $\beta_{s}$ & $\nu_{p}$ & $\beta_{p}$ & $a_{0}$ \\
\hline 0.7 & 0.485 & $2.5 \%$ & 0.25 & $0.0 \%$ & $0-0.15$ \\
\hline
\end{tabular}

Table 2: Dimensionless properties assumed for all cases used in this study.

pile barrier embedded in the soil layer over a rigid bedrock with the results obtained assuming the half space profile. The study starts with Case A, where a constant layer thickness is considered together with three pile aspect ratios for the elements of the barrier. Two of these aspect ratios correspond to piles that are shorter than the layer thickness, while the last ratio corresponds to the situation in which the piles reach the bedrock. For this case, the influence of the pile-soil stiffness ratio is also analysed. Only when the piles reach the rigid bedrock a significant variation of the barrier amplification ratio is obtained with respect to the half space scenario. After this finding, only configurations with piles having a length equal to the layer thickness are given further consideration. The influence of the layer thickness on the barrier behaviour is studied in Case B, together with the effects of changing the pile tip boundary condition at the interface between the piles and the rigid bedrock. Then, Case $\mathrm{C}$ studies the influence of the position of the external source with respect to the barrier, comparing the scenarios of the bedrock and half space soil profiles. In Cases D and E, the academic assumption of the single homogeneous layer over an infinitely rigid bedrock profile is generalized into two more realistic soil profiles: a homogeneous layer overlying a flexible bedrock (Case D), and a soil layer with a variablewith-depth properties over a rigid bedrock (Case E). Finally, several barrier configurations are analysed in Case $\mathrm{F}$ by changing the pile spacing or adding extra pile rows in order to confirm that the obtained results can be extrapolated to other disposition of the piles in the barrier. In the following, detailed comments of the different cases are presented.

\subsection{Case A. Influence of the pile dimensions}

Fig. 7 presents the average amplification ratio produced by the pile barrier as a function of the dimensionless frequency for the half space and bedrock profiles. The aspect ratios of the piles are indicated by the labels atop each column. For the bedrock profile, a layer thickness $H / d=20$ is assumed regardless of the pile aspect ratio. The natural frequencies of the layer (corresponding to $H=n \lambda_{s} / 4$ with $n=1,3,5 \ldots$ ) are marked with vertical dashed lines. Two different situations are found depending on whether or not the piles reach the rigid bedrock. When the piles are shorter than the thickness of the soil layer, the 
average amplification ratio obtained for the bedrock profile practically coincides with the one for the half space, showing a slight oscillation around it. For the studied frequency range, the barrier with the shortest piles $(L / d=5)$ provides virtually no reduction of the ground vibration; while the one formed by piles with $L / d=10$ only presents a small attenuation at high frequencies. For this last configuration in the bedrock profile, there is a frequency range $\left(a_{0}=0.04-0.08\right)$ in which the presence of the piles amplifies the average vertical displacements at the points located behind the barrier. On the other hand, for the case of the piles with a length equal to the layer thickness, a considerable change in the behaviour of the barrier is found between the bedrock and half space profiles. For the single layer over the rigid bedrock, the reduction of the pile barrier starts to increase from frequencies above the fundamental frequency of the soil layer, reaching the maximum reduction, i.e. minimum $\bar{A}_{r}$ value, around the second natural frequency $\left(H=3 \lambda_{s} / 4\right.$, i.e. $\left.a_{0}=0.0375\right)$. For higher frequencies, the barrier becomes less effective but continues to reduce the ground vibration at the points behind it $\left(\bar{A}_{r}<1\right)$. For the half space profile, the average amplification ratio of the barrier smoothly reduces as the frequency increases, reaching a minimum around $a_{0}=0.09$, and then starts to become less effective for higher frequencies but at a lower rate than in the case of the bedrock profile. Comparing the $\bar{A}_{r}$ ratios of the two soil types, it can be found that the largest reductions are found for the bedrock site around its second natural frequency, but for frequencies higher than the third natural frequencies the barrier becomes less effective in this medium compared with the half space.
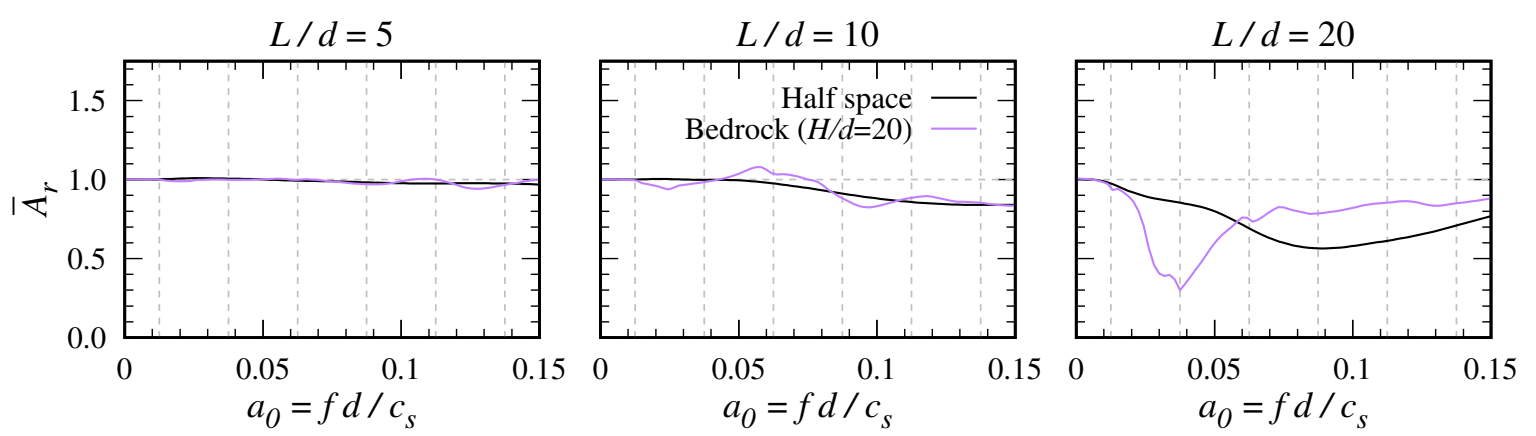

Figure 7: Case A, $E_{p} / E_{s}=250$. Average amplification ratio. Influence of the pile aspect ratio.

In order to explain why the performance of the pile barrier at high frequencies is reduced to a larger extent in the bedrock profile than in the half space domain for the $L / d=20$ piles, Fig. 8 shows, for the two studied profiles, the absolute value of the vertical displacements produced by the external source at certain observation points with and without considering the presence of the pile barriers. These observation points are located at the central line of the barrier $\left(y_{o} / d=0\right)$ and at a distance of $1 / 8\left(x_{o} / d=6\right)$, $1 / 4\left(x_{o} / d=12\right)$ and $1 / 2\left(x_{o} / d=24\right)$ of the width of the observation surface $(2 W / d)$. Expressing the results directly in terms of displacements gives a better explanation of the behaviour corresponding to the two soil profiles. For the half space, the displacements present an oscillatory but smooth behaviour with frequency, and the presence of the barrier only scales their value. On the contrary, for the bedrock profile, the variation of the soil response with frequency is different. Below the fundamental frequency 
of the soil, the energy introduced by the external load can not propagate through the layer so the displacements at the soil surface are limited. However, once this cut-off frequency is reached, the displacements of the soil rapidly increase, reaching values that can be three times higher than at the half space site. At higher frequencies, a highly frequency-dependent behaviour of the soil displacements is found. At these values of the dimensionless frequency $a_{0}$, the presence of the pile barrier in the bedrock profile modifies the variation with frequency of the soil displacements, shifting the curves toward higher frequencies. This shift, produced by the interference of different waves, is more evident for points more distant to the barrier: e.g., the minimum displacement for the point at $x_{o} / d=6$ is moved from $a_{0}=0.065$ to $a_{0}=0.07$ when the piles are included; but the shift of minimum value for $x_{o} / d=24$ goes from $a_{0}=0.12$ to $a_{0}=0.14$ if the barrier is present. At high frequencies, these shifts lead to an oscillation of the amplification ratios, as illustrated by the bottom row of Fig. 8, Therefore, the average reduction produced by the barrier in the bedrock profile is less effective for frequencies in which these shifts occur $\left(a_{0}>0.06\right.$ for the studied configuration).
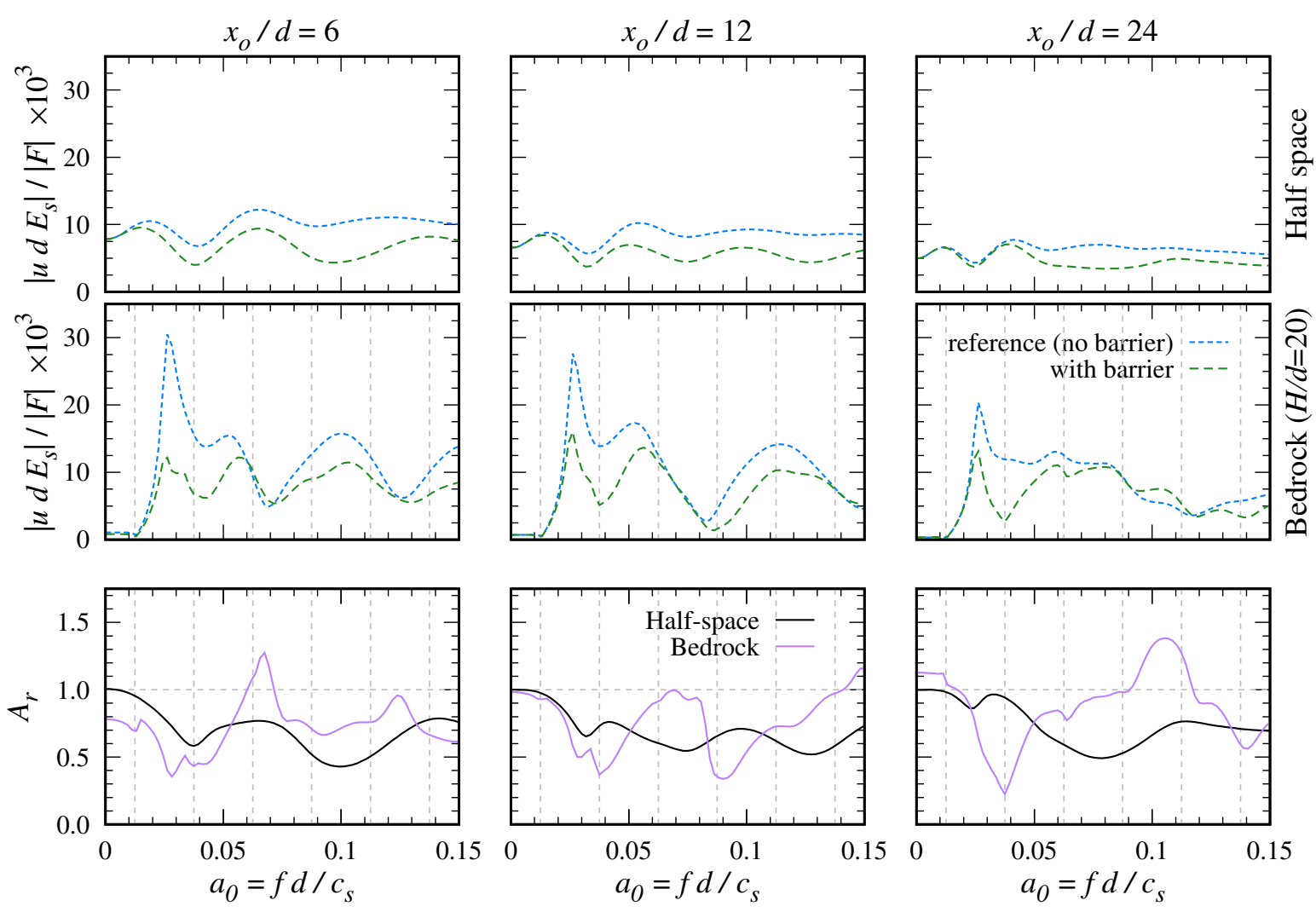

Figure 8: Case A, $E_{p} / E_{s}=250$. Amplitude of the dimensionless soil vertical displacements and amplification ratio at specific observation points located at the central line $\left(y_{o} / d=0\right)$ behind the barrier. Pile aspect ratio $L / d=20$.

In order to study the influence of the pile dimensions on the effectiveness of the barrier, Fig. 9 presents the amplitude of the soil displacements with and without the pile barrier and the corresponding amplification ratios for several frequencies and pile dimensions. The two first rows present the results at the second natural frequency of the layer profile 
for the configuration where the piles reach the rigid stratum. In the case of the bedrock profile, a clear attenuation of the vertical displacements of the points beyond the pile barrier is observed, while for the half space domain this reduction is not so important. The third and fourth rows show similar results but for a higher frequency $\left(a_{0}=0.1\right)$. For the bedrock profile, the previously observed interference between the different waves can be seen. The zone with minimum vertical displacements is slightly shifted close to the barrier (dashed lines could be used as reference). This leads to a slight amplification of the soil displacements in a zone immediately behind the barrier. However, this amplification should be understood as a local effect (due to the small reference displacements) rather than a significant increase in the magnitude of the soil displacements due to the barrier. These results also show the influence of the position of receiver point on the barrier performance. Finally, the fifth and sixth rows present the results for the barrier with $L / d=10$ piles for $a_{0}=0.1$. For this configuration, the influence of the bedrock is small.

Finally, Fig. 10 shows the results for Case A that are obtained by assuming different values of the pile-soil stiffness ratio. The initial value $E_{p} / E_{s}=250$ was considered as representative of concrete piles $\left(E_{p} \approx 30 \mathrm{MPa}\right)$ embedded in a medium-soft soil $\left(c_{s} \approx 150\right.$ $\mathrm{m} / \mathrm{s})$. Cases with different soil or pile properties are simulated by changing the value of the $E_{p} / E_{s}$ ratio. The obtained results show that the behaviour of the barrier is similar regardless of the pile-soil stiffness ratio. Furthermore, for configurations with $L / d \leq 10$ the average amplification ratios virtually coincide for $E_{p} / E_{s}=100-500$. On the other hand, for the barrier with the most slender piles $L / d=20$, a larger pile-soil stiffness ratio improves the performance of the barrier. The influence of the stiffness ratio $E_{p} / E_{s}$ is observed at high frequencies for the half space domain, while it is more significant around the second natural frequency for the bedrock profile.

\subsection{Case B. Influence of layer thickness and pile tip boundary conditions}

From the results presented in Figs. 7 and 10 it can be concluded that the behaviour of the pile barrier is only significantly altered by the presence of the rigid bedrock if the piles reach it. For this reason, and in order to study the effects of the layer thickness, Fig. 11 compares the average amplification ratio obtained for the half space profile with the one obtained assuming a bedrock profile in which the layer thickness coincides with the pile aspect ratio. The configuration with short piles $L / d=5$ is omitted as it would imply unrealistic short layers for typical pile dimensions. Note that, as the layer thickness changes, the natural frequencies of the soil profile (indicated by the vertical dashed lines) do not coincide in terms of $a_{0}$ for the cases of $L / d=10$ and 20. For the bedrock profile, the influence of the pile-bedrock interface conditions is investigated as well. Free, hinged, and fixed tip conditions are considered. The importance of the pile tip condition is found to be negligible, however. The results for free and hinged tips practically coincide, while the differences between these and the fixed condition are only significant at low frequencies, in particular for the shortest piles. For the fixed tip condition, a local minimum is observed at the layer fundamental frequency. This effect is produced because the pile deformation is not compatible with the soil displacements corresponding to the layer fundamental mode, but it is not really important as for the bedrock profile the displacements on the 

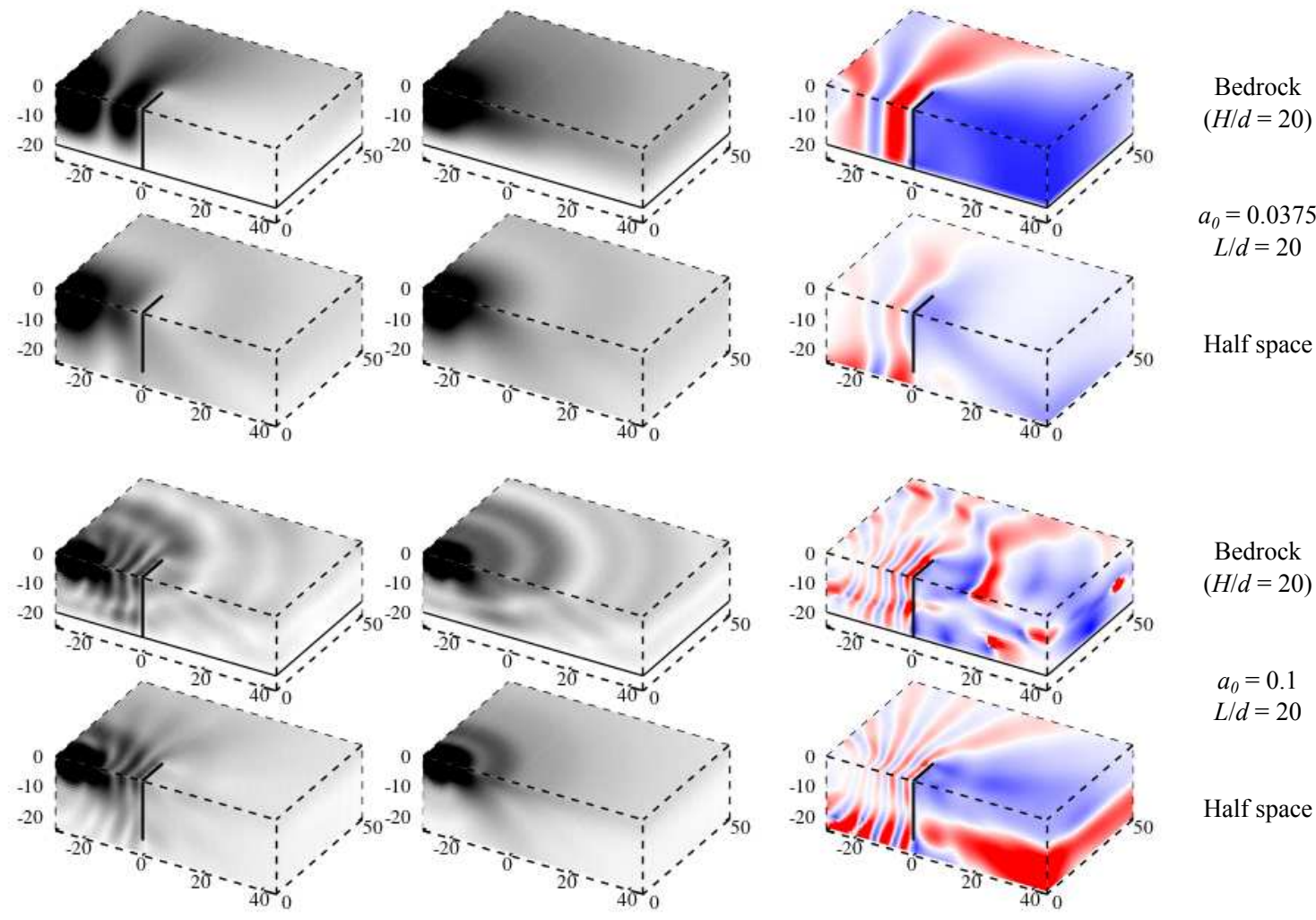

\section{Bedrock}

$(H / d=20)$

$a_{0}=0.1$

$L / d=20$

Half space
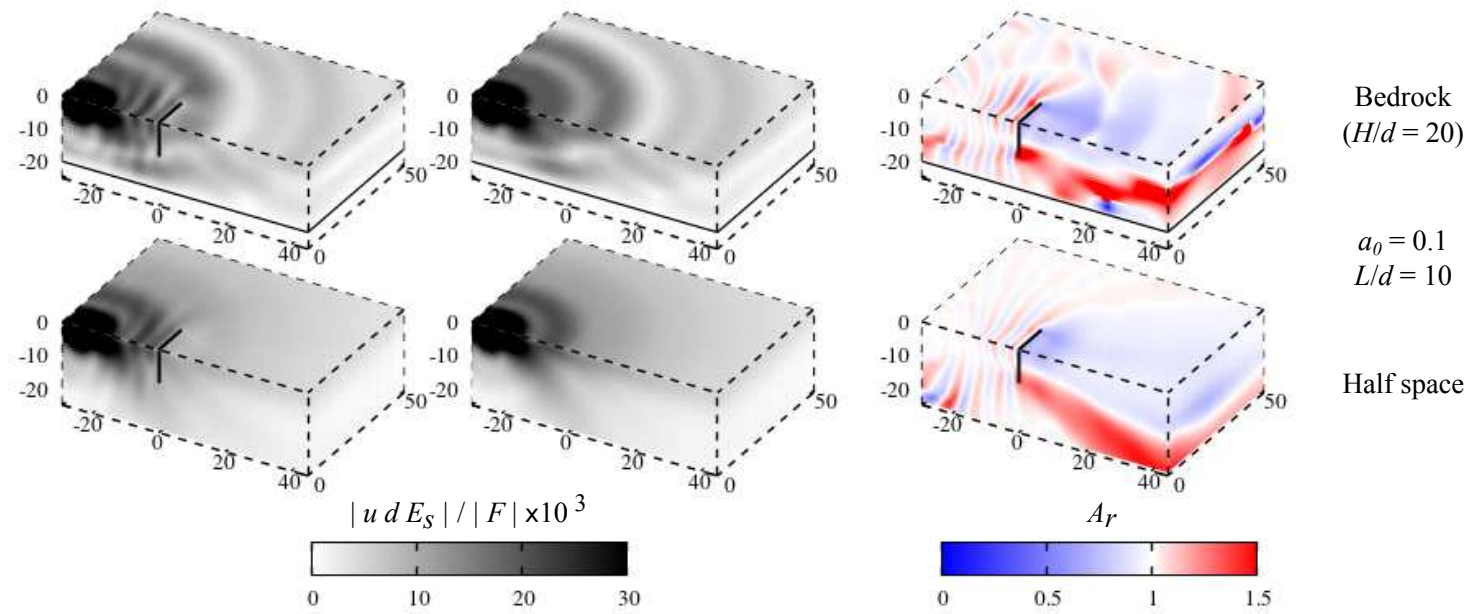

Figure 9: Case A, $E_{p} / E s=250$. Grey scale: Amplitude of dimensionless soil vertical displacements with (left) and without (right) the presence of the pile barrier. Color scale: Amplification ratio. Comparison between the half space (bottom) and bedrock with thickness $H / d=20$ (top) profiles. Various pile aspect ratios $L / d$ and dimensionless excitation frequencies $a_{0}$. Geometry of the soil domain expressed in terms of pile diameter $d$.

surface produced by the external source are quite small at these frequencies, as shown in Fig. 8, Regarding the influence of the layer thickness, the same trend can be seen for the two studied configurations: the maximum reduction takes place around the second natural frequency of the soil layer, and the value of the $\bar{A}_{r}$ ratio increases for larger 

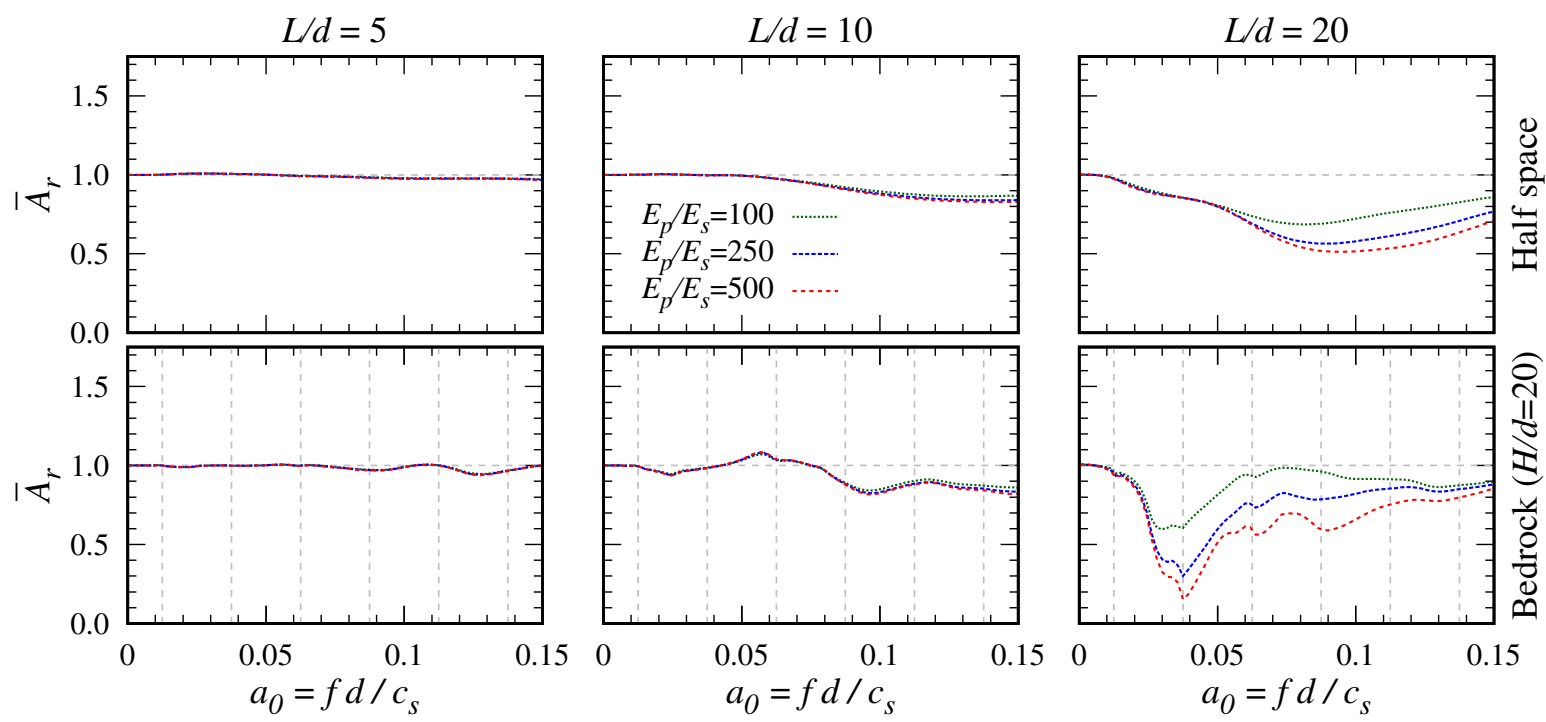

Figure 10: Case A. Average amplification ratio. Influence of the pile-soil stiffness ratio.

excitation frequencies. However, it is important to highlight that, in the studied range, the difference in the minimum value of the average reduction factor depending on the soil profile is higher for the shortest piles as it goes from $\bar{A}_{r}=0.8$ for the half space to $\bar{A}_{r}=0.4$ for the bedrock profile (for the $L / d=20$ configuration, these minimum values are 0.55 and 0.3 respectively).
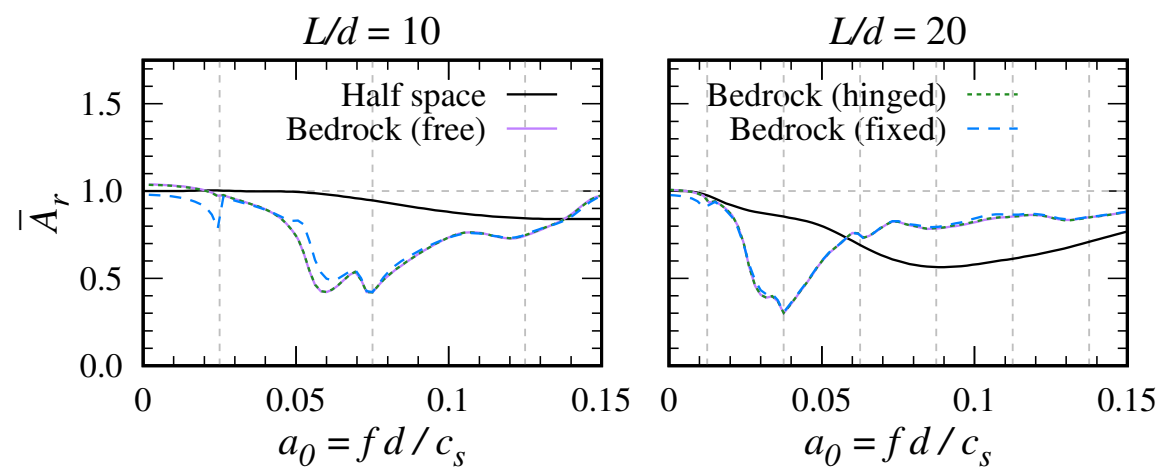

Figure 11: Case B. Average amplification ratio. Influence of the layer thickness and pile tip boundary condition.

\subsection{Case C. Influence of the external source position}

The influence of the position of the external source on the barrier performance is studied in Fig. 12, which presents the average amplification ratio for the half space and bedrock profiles as functions of this parameter. Note that the distance of the source with respect to the barrier is presented in terms of $r_{s} / \lambda_{s}$ instead of $r_{s} / d$ in order to obtain similar trends for the three studied frequencies. These frequencies correspond to the second and third natural frequencies of the soil layer $\left(H / \lambda_{s}=3 / 4,5 / 4\right)$, plus the intermediate value 
$\left(H / \lambda_{s}=1\right)$. The point marker indicates the results corresponding to the source position $\left(r_{s} / d=24\right)$ assumed in the other cases. The results depend significantly on the soil profile. For the half space, large reductions are obtained when the load is placed near the barrier $\left(r_{s} / \lambda_{s}<1\right)$. For larger separations, the average attenuation ratio of the barrier increases up to a limit value which remains constant regardless of the source separation distance. On the contrary, for the bedrock profile, the effectiveness of the pile barrier is maintained for all of the studied positions of the external load. The average amplification ratio oscillates depending on $r_{s} / \lambda_{s}$, but does not increase as the source moves away from the barrier. In the case where the ratio $H / \lambda_{s}=5 / 4$, the efficiency of the pile barrier can be larger in the half space domain (close loads) or in the bedrock site (distant loads), depending on the source position. The influence of the source position on the performance can be explained considering the different ways in which the energy is transmitted inside the two studied soil media. In the case of the bedrock profile, the propagation of the energy is channelled through the soft layer. On the other hand, for the half space domain, only part of the energy introduced by the external load is transmitted through surface waves and the rest propagates through body waves into the half space (as shown in Fig. 9). In this case, as the source moves away from the barrier, only the part which is transmitted through surface waves reaches the barrier and, therefore, can be attenuated.
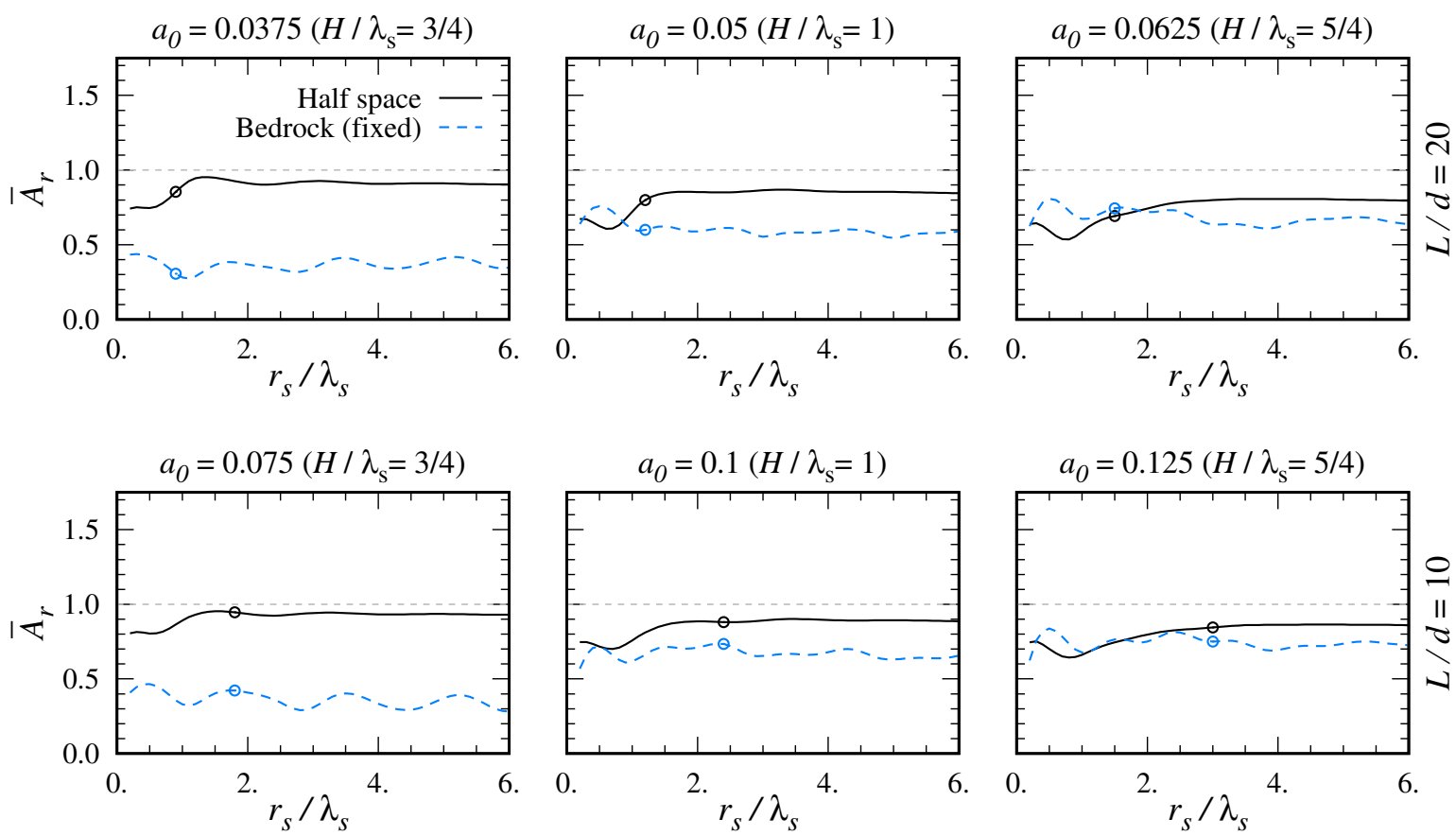

Figure 12: Case C. Average amplification ratio. Influence of the position of the source. Point markers indicate the source position used in other cases $\left(r_{s} / d=24\right)$.

\subsection{Case D. Influence of the stiffness of the lower stratum}

In the previous analyses, the case of an infinitely rigid bedrock has been considered. However, in reality, the lower medium has a finite stiffness. To study the influence of the stiffness of the bedrock upon the barrier performance, Fig. 13 presents the average 
amplification obtained by assuming different $E_{b} / E_{s}$ values. Free-tip pile-soil interface conditions are assumed for all of the soil profiles. The results show that the largest reductions are still found for the rigid bedrock case. However, noticeable differences with respect to the half space profile $\left(E_{b} / E_{s}=1\right)$ are observed for the other layered media considered here. When the lower layer is assumed to be flexible, the minimum value of the average amplification ratio is shifted from the second natural frequency of the layer over rigid base towards lower frequencies. At higher frequencies, the average amplification ratio $\bar{A}_{r}$ of all layered profiles increases, reaching values above the one corresponding to the half space, as well as the perfect bedrock case. When the pile aspect ratio has a value of 20, the average amplification ratio in the flexible bedrock case is again reduced at high frequencies, obtaining larger barrier effectiveness than for the perfect bedrock case.
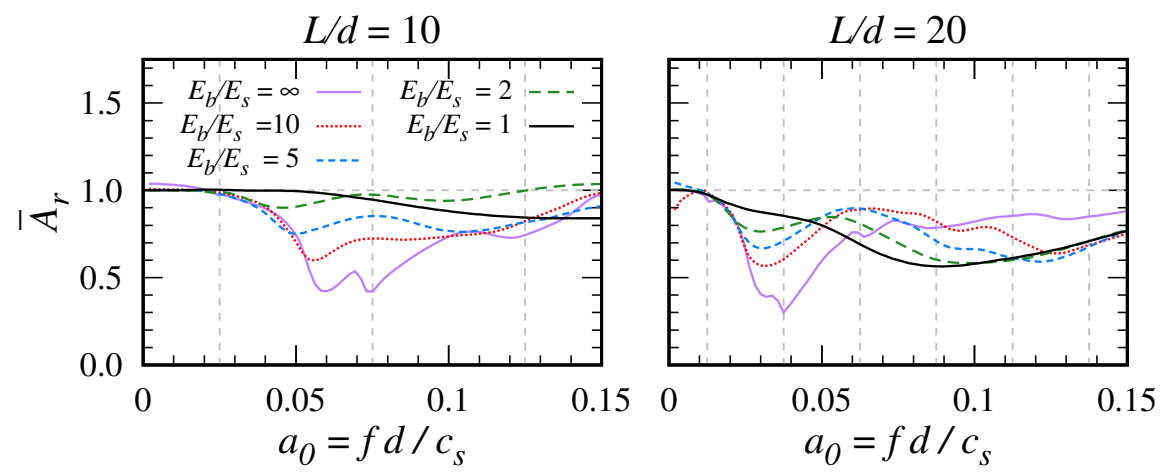

Figure 13: Case D. Average amplification ratio. Influence of the flexibility of the bedrock.

\subsection{Case E. Influence of the profile of the soil layer}

In consolidated soils, the shear wave velocity increases with depth in a continuous way. In order to check how the assumption of a variable profile for the soft layer changes the obtained results, Fig. 14 presents the average amplification ratio for three different scenarios. First, the previous case of a homogeneous layer over an infinitely rigid bedrock is considered. Then, two profiles in which the soil Young's modulus linearly increases with depth (Gibson soil) are assumed. These profiles are determined by the value of the Young's modulus at the free-surface level (either $0.5 E_{s}$ or $0.25 E_{s}$ ), and are defined in order to present the same average $E_{p} / E_{s}$ ratio along the layer thickness as the homogeneous medium:

$$
\left\{\begin{aligned}
0.25 E_{s}: & E_{s}(z)=[0.25+1.75(z / L)]\left(E_{p} / 250\right) \\
0.5 E_{s}: & E_{s}(z)=[0.5+1.5(z / L)]\left(E_{p} / 250\right)
\end{aligned}\right.
$$

The shear wave velocity used to compute the dimensionless frequency is the one that corresponds to the average value of $E_{s}=E_{p} / 250$. The other soil parameters $\left(\rho_{s}, \nu_{s}, \beta_{s}\right)$ are kept the same for the whole profile. Note that the vertical dashed lines in Fig. 14 indicate the natural frequencies of the homogeneous layer, which do not coincide with the natural frequencies of the variable profiles. Indeed, a small shift towards lower frequencies can be seen in the minimum value of $\bar{A}_{r}$ due to the reduction of the second natural frequency of the variable soil layer compared to an uniform layer with the same average stiffness 
[49]. Despite this small shift and the fact that the frequency dependence of the average amplification ratio increases for the two variable profiles, the same trend is observed as in the case of the homogeneous layer.
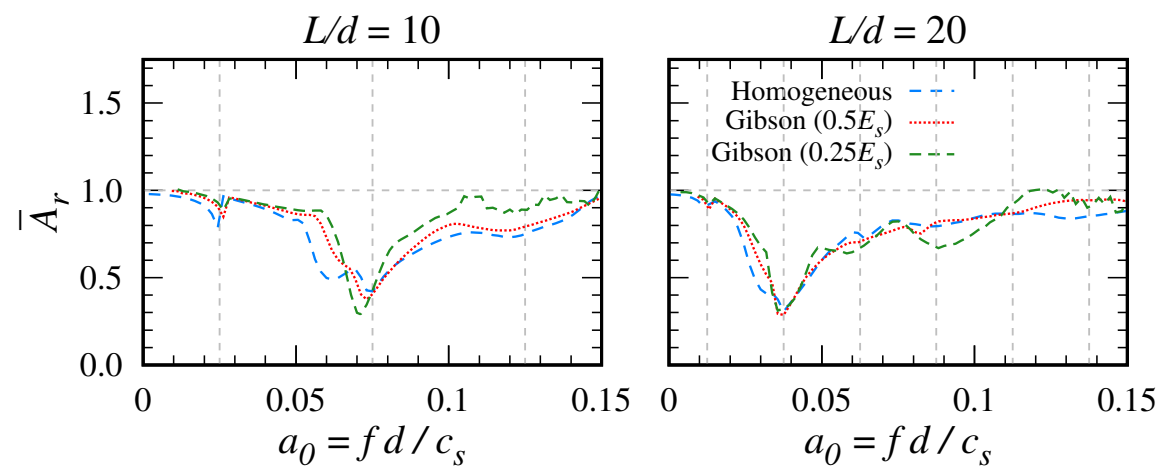

Figure 14: Case E. Average amplification ratio. Influence of the profile of the soil layer.

\subsection{Case F. Influence of the pile barrier configuration}

Finally, in order to check if the previous results can be extrapolated to other pile barrier configurations, the pile dispositions presented in Fig. 15] are considered. The same barrier width is kept for all configurations to preserve the same observation surface. Thus, the pile separation is changed by introducing or removing some piles. Also, two configurations formed by a double pile barrier are included. In those configurations, labelled as double, the $s / d$ ratio represents the distance between the projections over the $y$ axis of two consecutive piles, and a fixed separation between the two rows $s_{x} / d=2$ is assumed. The average amplification ratios for the half space and bedrock profiles for these five configurations are presented in Fig. 16. As expected, the smaller the spacing between the piles, the larger the reduction produced by the barrier. This increase in the barrier performance is found from medium-high frequencies $\left(a_{0}>0.05\right)$ for the half space profile, and above the fundamental frequency of the soil layer for the bedrock site. For the latter soil type, the importance of the pile separation is the same regardless of the pile aspect ratio, while for the half space domain the magnitude of the influence of the $s / d$ ratio is significantly higher for slender piles. The results for the configurations with double pile rows coincide with the ones of the line configurations with the same $s / d$ for $a_{0}<0.05$. For higher frequencies, different results are found depending on the problem. For the half space profile, larger reductions are obtained if the piles are distributed into two rows for the configurations $s / d=2$, but the opposite effect is seen for the $s / d=4$ barriers. For the bedrock profile, on the other hand, the effectiveness of a barrier composed of aligned piles is always higher than for configurations with two rows. Despite these differences, it can be seen that the main trends of the average amplification ratios for the half space and bedrock profile remain the same for all of the studied pile configurations. 


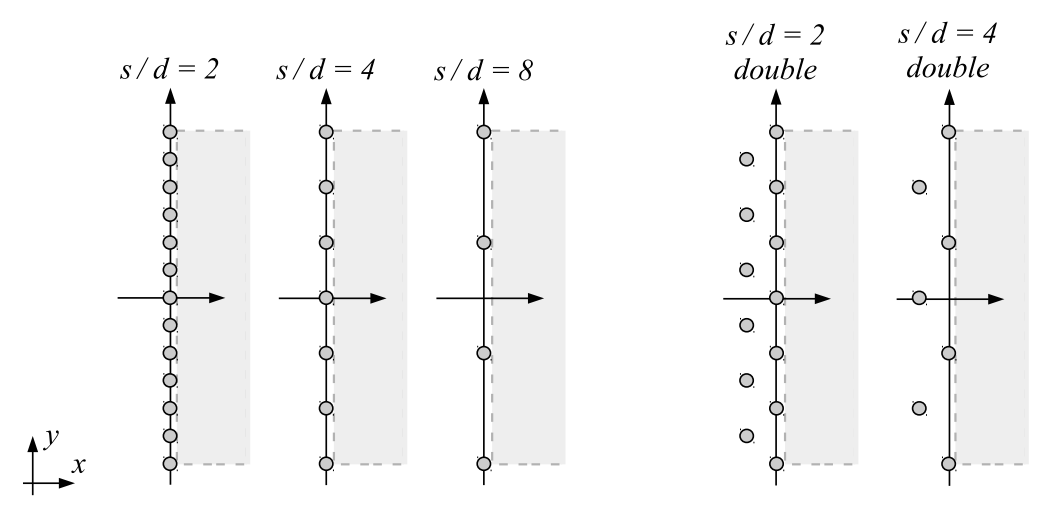

Figure 15: Pile barrier configurations analysed in Case F.
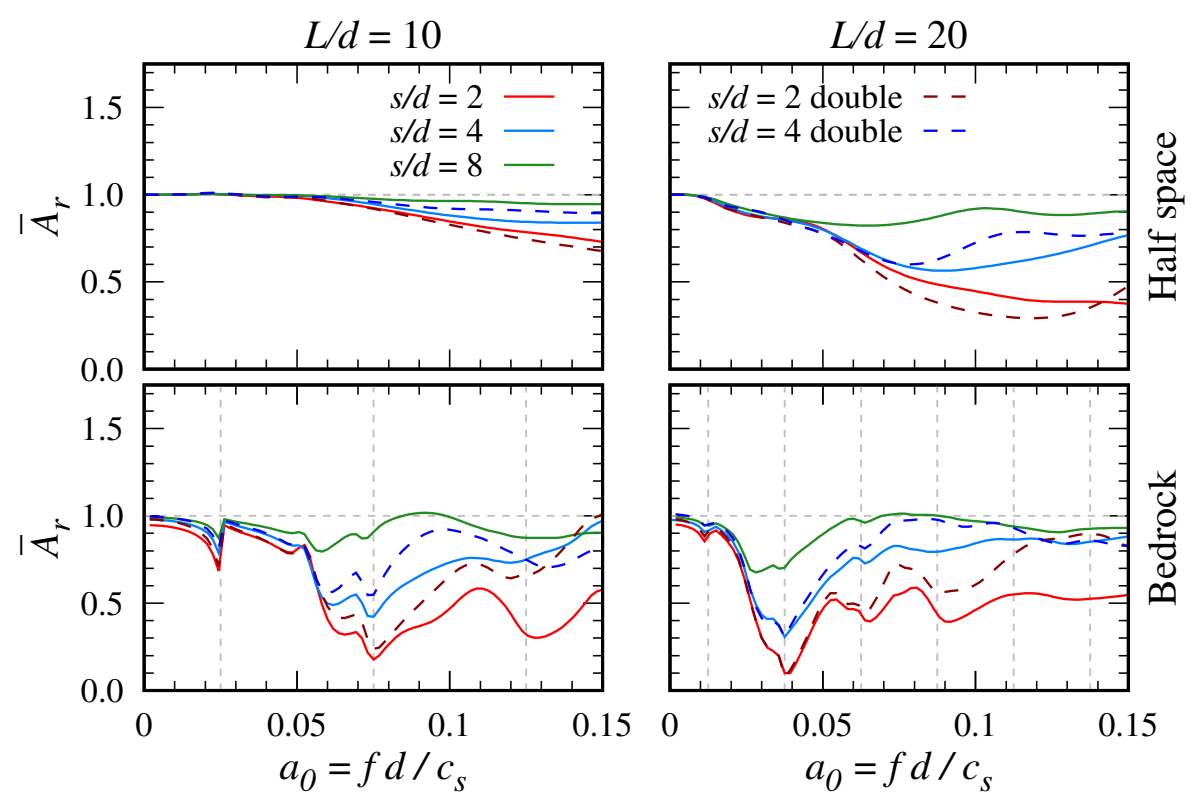

Figure 16: Case F. Average amplification ratio. Influence of the pile separation and pile disposition.

\section{Conclusions}

In this work, the performance of a pile barrier as ground vibration mitigation measure is analysed through a simplified integral model. In the proposed formulation, the soil behaviour is obtained making use of the reciprocity theorem in elastodynamics and Green's functions for the layered half space. The piles are modelled as unidimensional Timoshenko beam elements, are treated as load lines acting within the layered soil domain. The range of validity of the integral model is determined by benchmarking against a Boundary Element formulation. From this validation, some conclusions can be drawn:

- The integral model accurately reproduces the response obtained by the BE model for dimensionless frequencies below a limit value. In this frequency range, the wavelength in the soil is large compared to the dimensions of the barrier, so that diffraction effects are small. At higher frequencies, the results of the integral model still 
follow similar trends as the ones of the BE model.

- This limit frequency value is strongly influenced by the distance between the piles in the barrier, with a larger spacing between the piles leading to an increased range of validity of the integral model.

- The accuracy and high computational performance of the integral model makes it a suitable tool for preliminary designs or the optimization of pile barriers.

The integral model is subsequently used in order to study how the performance of the pile barrier, measured in terms of the average amplification ratio, changes depending on the considered soil profile. For this purpose, the results for the half space are compared to the ones of a single layer over a rigid bedrock profile. The main conclusions obtained from the analyses are:

- Appreciable differences are found between the half space and bedrock profiles only if the piles reach the rigid stratum. Otherwise, the results obtained for the bedrock site oscillate around those for the half space domain. For the configurations in which the piles reach the rigid bedrock, the influence of the pile-bedrock interface condition is almost negligible.

- The highest reduction due to the presence of the barrier in the bedrock profile is produced at frequencies around the second natural frequency of the soil layer $\left(H=3 \lambda_{s} / 4\right)$.

- For higher frequencies, a decrement in the barrier efficiency is produced due to the shifts of the soil displacement fields with and without the barrier produced by the interference of the different waves. In this situation, a high dependence of the performance of the barrier on the position of the receiver point is found.

- The performance of the pile barrier in the half space is highly reduced if the excitation load is placed at a dimensionless distance $r_{s} / \lambda_{s}>1$ from the pile barrier. On the contrary, the average amplitude reduction produced by the barrier in the bedrock profile oscillates around the same mean value regardless of the position of the source.

- The effect of the presence of the infinitely rigid bedrock is smaller when the finite stiffness of the underlying stiffer half space is considered. However, the trend in the barrier performance remains similar for the flexible bedrock profiles. Considering a continuous variation with depth of the properties of the upper layer does not alter the main effects produced by the presence of the rigid bedrock.

- Pile barrier configurations with closer piles produce, as expected, larger attenuations of the vertical ground motion. The influence of the pile spacing is higher for the bedrock profile or for configurations with slender piles embedded in a half space domain. A better performance of the pile barrier in the bedrock profile is found when the piles are distributed along one single row. 


\section{Acknowledgments}

The authors affiliated to Universidad de Las Palmas de Gran Canaria are grateful for the support from Ministerio de Economía, Industria y Competitividad (MINECO) and Agencia Estatal de Investigación (AEI) of Spain and FEDER through research project BIA2017-88770-R. Also, G.M. Álamo is recipient of FPU research fellowship FPU14/06115 from Ministerio de Educación, Cultura y Deporte of Spain, which also provided him with financial support (EST16/00617) for a short-term research stay at KU Leuven.

\section{References}

[1] Richart Jr., F.E., Hall, J.R., Woods, R.D.. Vibrations of Soils and Foundations. Englewood Cliffs, NJ: Prentice-Hall; 1970.

[2] Woods, R.D.. Screening of surface waves in soils. Journal of the Soil Mechanics and Foundations Division, ASCE 1968;94(4):951-979.

[3] Alzawi, A., Hesham El Naggar, M.. Full scale experimental study on vibration scattering using open and in-filled (GeoFoam) wave barriers. Soil Dynamics and Earthquake Engineering 2011;31(3):306-317.

[4] Coulier, P., Hunt, H.E.M.. Experimental study of a stiff wave barrier in gelatine. Soil Dynamics and Earthquake Engineering 2014;66:459-463.

[5] Coulier, P., Cuéllar, V., Degrande, G., Lombaert, G.. Experimental and numerical evaluation of the effectiveness of a stiff wave barrier in the soil. Soil Dynamics and Earthquake Engineering 2015;77:238-253.

[6] Dasgupta, B., Beskos, D., Vardoulakis, I.G.. Vibration isolation using open or filled trenches. Part 2: 3D homogeneous soil. Computational Mechanics 1990;6(2):129-142.

[7] Leung, K.L., Beskos, D.E., Vardoulakis, I.G.. Vibration isolation using open or filled trenches. Part 3: 2-D non-homogeneous soil. Computational Mechanics 1990;7(2):137-148.

[8] Andersen, L., Nielsen, S.R.. Reduction of ground vibration by means of barriers or soil improvement along a railway track. Soil Dynamics and Earthquake Engineering 2005;25(7-10):701-716.

[9] Andersen, L., Augustesen, A.. Mitigation of traffic-induced ground vibration by inclined wave barriers: a three-dimensional numerical analysis. In: Proceedings of the 16th International Congress on Sound and Vibration. 2009,.

[10] Thompson, D.J., Jiang, J., Toward, M.G., Hussein, M.F., Ntotsios, E., Dijckmans, A., et al. Reducing railway-induced ground-borne vibration by using open trenches and soft-filled barriers. Soil Dynamics and Earthquake Engineering 2016;88:45-59.

[11] Persson, P., Persson, K., Sandberg, G.. Numerical study of reduction in ground vibrations by using barriers. Engineering Structures 2016;115:18-27. 
[12] Van hoorickx, C., Sigmund, O., Schevenels, M., Lazarov, B.S., Lombaert, G.. Topology optimization of two-dimensional elastic wave barriers. Journal of Sound and Vibration 2016;376:95-111.

[13] Bordón, J.D.R., Aznárez, J.J., Maeso, O.. Two-dimensional numerical approach for the vibration isolation analysis of thin walled wave barriers in poroelastic soils. Computers and Geotechnics 2016;71:168-179.

[14] Andersen, L., Liingaard, M.. Vibration screening with sheet pile walls. In: Environmental Vibrations: Prediction, Monitoring, Mitigation and Evaluation (ISEV 2005). Spon press; 2005, p. 429-437.

[15] Andersen, L., Frigaard, P., Augustesen, A.. Mitigation of ground vibration by double sheet-pile walls. In: Eren, Ö., Mohamed, A., Günyakti, A., Soyer, E., Bilsel, H., Kunt, M., editors. Proceedings of the 8th International Congress on Advances in Civil Engineering (ACE 2008); vol. 2. Eastern Mediterranean University Press; 2008, p. 247-254.

[16] Dijckmans, A., Ekblad, A., Smekal, A., Degrande, G., Lombaert, G.. Efficacy of a sheet pile wall as a wave barrier for railway induced ground vibration. Soil Dynamics and Earthquake Engineering 2016;84:55-69.

[17] Van hoorickx, C., Schevenels, M., Lombaert, G.. Double wall barriers for the reduction of ground vibration transmission. Soil Dynamics and Earthquake Engineering 2017;97:1-13.

[18] Bordón, J.D.R., Van hoorickx, C., Aznárez, J.J., Schevenels, M., Maeso, O., Lombaert, G.. Shape optimized inclined single and double wall wave barriers for ground vibration mitigation. Soil Dynamics and Earthquake Engineering 2018;112:215-231.

[19] Woods, R.D., Barnett, N.E., Sagesset, R.. Holography - a new tool for soil dynamics. Journal of Geotechnical Engineering 1974;100(11):1231-1247.

[20] Liao, S., Sangrey, D.A.. Use of piles as isolation barriers. Journal of Geotechnical Engineering 1978;104(9):1139-1152.

[21] Avilés, J., Sánchez-Sesma, F.J.. Piles as barriers for elastic waves. Journal of Geotechnical Engineering 1983;109(9):1133-1146.

[22] Avilés, J., Sánchez-Sesma, F.J.. Foundation isolation from vibrations using piles as barriers. Journal of Engineering Mechanics 1988;114(11):1854-1870.

[23] Kattis, S.E., Polyzos, D., Beskos, D.E.. Vibration isolation by a row of piles using a 3-D frequency domain BEM. International Journal for Numerical Methods in Engineering 1999;46(5):713-728.

[24] Kattis, S.E., Polyzos, D., Beskos, D.E.. Modelling of pile wave barriers by effective trenches and their screening effectiveness. Soil Dynamics and Earthquake Engineering 1999;18(1):1-10. 
[25] Tsai, P.H., Feng, Z.Y., Jen, T.l.. Three-dimensional analysis of the screening effectiveness of hollow pile barriers for foundation-induced vertical vibration. Computers and Geotechnics 2008;35(3):489-499.

[26] Gao, G.Y., Li, Z.Y., Qiu, C., Yue, Z.Q.. Three-dimensional analysis of rows of piles as passive barriers for ground vibration isolation. Soil Dynamics and Earthquake Engineering 2006;26(11):1015-1027.

[27] Xia, T.D., Sun, M.M., Chen, C., Chen, W.Y., Ping, X.. Analysis on multiple scattering by an arbitrary configuration of piles as barriers for vibration isolation. Soil Dynamics and Earthquake Engineering 2011;31(3):535-545.

[28] Huang, J., Shi, Z.. Attenuation zones of periodic pile barriers and its application in vibration reduction for plane waves. Journal of Sound and Vibration 2013;332(19):4423-4439.

[29] Liu, X., Shi, Z., Xiang, H., Mo, Y.L.. Attenuation zones of periodic pile barriers with initial stress. Soil Dynamics and Earthquake Engineering 2015;77:381-390.

[30] Liu, X., Shi, Z., Mo, Y.L.. Comparison of 2D and 3D models for numerical simulation of vibration reduction by periodic pile barriers. Soil Dynamics and Earthquake Engineering 2015;79:104-107.

[31] Cai, Y.Q., Ding, G.Y., Xu, C.J.. Amplitude reduction of elastic waves by a row of piles in poroelastic soil. Computers and Geotechnics 2009;36(3):463-473.

[32] Xu, B., Lu, J.F., Wang, J.H.. Numerical analysis of the isolation of the vibration due to Rayleigh waves by using pile rows in the poroelastic medium. Archive of Applied Mechanics 2010;80(2):123-142.

[33] $\mathrm{Xu}, \mathrm{P} .$. Analysis of isolation effectiveness of shear waves by a row of hollow pipe piles in saturated soils. Transport in Porous Media 2017;120(2):415-432.

[34] Cacciola, P., Espinosa, M.G., Tombari, A.. Vibration control of piled-structures through structure-soil-structure-interaction. Soil Dynamics and Earthquake Engineering 2015;77:47-57.

[35] Tombari, A., Garcia Espinosa, M., Alexander, N.A., Cacciola, P.. Vibration control of a cluster of buildings through the Vibrating Barrier. Mechanical Systems and Signal Processing 2018;101:219-236.

[36] Brûlé, S., Javelaud, E.H., Enoch, S., Guenneau, S.. Experiments on seismic metamaterials: Molding surface waves. Physical Review Letters 2013;112:133901.

[37] Finocchio, G., Casablanca, O., Ricciardi, G., Alibrandi, U., Garesc, F., Chiappini, M., et al. Seismic metamaterials based on isochronous mechanical oscillators. Applied Physics Letters 2014;104:191903.

[38] Krödel, S., Thomé, N., Daraio, C.. Wide band-gap seismic metastructures. Extreme Mechanics Letters 2015;4:111-117. 
[39] Palermo, A., Krödel, S., Marzani, A., Daraio, C.. Engineered metabarrier as shield from seismic surface waves. Scientific Reports 2016;6:39356.

[40] Colombi, A., Roux, P., Guenneau, S., Gueguen, P., Craster, R.V.. Forests as a natural seismic metamaterial: Rayleigh wave bandgaps induced by local resonances. Scientific Reports 2016;6:19238.

[41] Colombi, A., Guenneau, S., Roux, P., Craster, R.V.. Transformation seismology: Composite soil lenses for steering surface elastic Rayleigh waves. Scientific Reports 2016;6:25320.

[42] Colombi, A., Colquitt, D., Roux, P., Guenneau, S., Craster, R.V.. A seismic metamaterial: The resonant metawedge. Scientific Reports 2016;6:27717.

[43] Ai, Z.Y., Cao, Z.. Vibration isolation of row of piles embedded in transverse isotropic multi-layered soils. Computers and Geotechnics 2018;99:115-129.

[44] Álamo, G.M., Martínez-Castro, A.E., Padrón, L.A., Aznárez, J.J., Gallego, R., Maeso, O.. Efficient numerical model for the computation of impedance functions of inclined pile groups in layered soils. Engineering Structures 2016;126:379-390.

[45] Pak, R.Y.S., Guzina, B.B.. Three-dimensional Green's functions for a multilayered half-space in displacement potentials. Journal of Engineering Mechanics, ASCE 2002;128(4):449-461.

[46] Friedman, Z., Kosmatka, J.. An improved two-node Timoshenko beam finite element. Computers and Structures 1993;47(3):473-481.

[47] Maeso, O., Aznárez, J.J., García, F.. Dynamic impedances of piles and groups of piles in saturated soils. Computers and Structures 2005;83:769-782.

[48] Bordón, J.D.R., Aznárez, J.J., Maeso, O.. Dynamic model of open shell structures buried in poroelastic soils. Computational Mechanics 2017;60(2):269-288.

[49] Rovithis, E.N., Parashakis, H., Mylonakis, G.E.. 1D harmonic response of layered inhomogeneous soil: Analytical investigation. Soil Dynamics and Earthquake Engineering 2011;31(7):879-890. 